\title{
A New Monte Carlo Programme for Simulating Light Transport through Port Wine Stain Skin.
}

\section{Abstract}

A new Monte Carlo programme is presented for simulating light transport through

clinically normal skin and skin that has Port Wine Stains (PWS) vessels. The program consists of an eight layer mathematical skin model constructed from optical coefficients described previously.

A simulation including diffuse illumination at the surface and subsequent light transport through the model is carried out using a Radiative Transfer Theory (RTT) ray-tracing technique. Total reflectance values over 39 wavelengths are scored by the addition of simulated light returning to the surface within a specified region and surface reflections (calculated using Fresnel's equations). These reflectance values are compared to measurements from individual participants and characteristics of the model are adjusted until adequate agreement is produced between simulated and measured skin reflectance curves.

The absorption and scattering coefficients of the epidermis are adjusted through changes in the simulated concentrations and mean diameters of epidermal melanosomes to reproduce non-lesional skin colour. Pseudo-cylindrical horizontal vessels are added to the skin model and their simulated mean depths, diameters and number densities are adjusted to reproduce measured PWS skin colour.

Accurate reproductions of colour measurement data are produced by the program, resulting in realistic predictions of melanin and PWS blood vessel parameters. Using a modest personal computer, the simulation currently requires an average of five and a half days to complete. 


\section{Introduction}

Port Wine Stains (PWS) are the most common of all congenital vascular malformations. They are caused by a permanent swelling of blood vessels within the dermis and present as a red lesion on the skin. PWS often constitute a significant disfigurement and can result in major psychological problems. Thus, many people who suffer with PWS seek treatment.

Laser treatment is currently the therapy of choice for PWS. The object of treatment is to selectively heat the swollen blood vessels, which constitute the lesion, resulting in irreversible damage and subsequent removal of these vessels. Selectivity may be achieved through careful choice of treatment parameters including wavelength, pulse duration and energy per pulse to match the depths, diameters and number density of vessels within a lesion.

Approximately half of patients who undergo laser treatment for PWS respond poorly or not at all. One reason for this poor response is that the laser parameters used are virtually identical for all PWS patients despite the availability of laser systems with user specific settings $[1,2]$. In order to optimise laser settings for each individual patient, the practitioner must be provided with information regarding the vascular architecture from each patient, such as the depths, diameters and number density of PWS vessels.

Historically, such information has been made available from some patients through punch biopsies. However, such a technique is subject to distortion in the sample and is likely to result in a scar, which would contribute further to the disfigurement of the lesion, thus rendering it unacceptable for the majority of patients. Imaging methods designed to provide 'virtual biopsies', such as ultrasound imaging or Optical Coherence Tomography, may one day provide a non-invasive alternative. However, due to the need for high resolution and imaging depths of $3-4 \mathrm{~mm}$, the devices currently available to the practitioner do not provide adequate information in the clinical setting.

Biopsy studies $[3,4]$ have shown that skin colour is affected by the depths, diameters and number density of PWS vessels. Although, skin colour measurements are the most established and widely used technique for the objective assessment of PWS skin [5-7], to 
date this method has not been used to extract such information. In this paper, a new Monte Carlo simulation is presented which demonstrates the capability of using skin colour measurements to predict the vessel parameters of an individual PWS lesion.

\section{Methods}

\section{Skin Model}

An eight layer skin model was created in 3 dimensional Cartesian space. The dimensions and chromophore concentrations of the model layers were based upon the work of Meglinski and Matcher [8], whose parameters are in agreement with experimental studies (for example, [9-11]). The layer representing the living epidermis in Meglinski and Matcher's work was divided into two layers in the new programme in order to facilitate differences in melanin properties (Table 1). Estimations of representative melanosome concentrations and sizes were based upon an analysis of the literature [11-16].

Table 1: Summary of layer dimensions and initial chromophore content; values derived from * [8] and ${ }^{+}[14]$.

\begin{tabular}{|c|c|c|c|c|}
\hline Layer & $\begin{array}{c}\text { Thickness } \\
(\mu \mathrm{m})^{*}\end{array}$ & 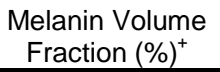 & $\begin{array}{l}\text { Mean Melanin Granule } \\
\text { Diameter }(\mathrm{nm})^{+}\end{array}$ & $\begin{array}{l}\text { Blood Volume } \\
\text { Fraction }(\%)^{*}\end{array}$ \\
\hline Stratum Corneum & 20 & 0.1 & 5 & 0 \\
\hline Superficial Epidermis & 30 & 1.0 & 50 & 0 \\
\hline Deep Epidermis & 50 & 2.0 & 100 & 0 \\
\hline Papillary Dermis & 150 & 0 & $\mathrm{n} / \mathrm{a}$ & 4 \\
\hline Upper Vascular Plexus & 80 & 0 & $\mathrm{n} / \mathrm{a}$ & 30 \\
\hline Reticular Dermis & 1500 & 0 & $\mathrm{n} / \mathrm{a}$ & 4 \\
\hline Deep Vascular Plexus & 80 & 0 & $n / a$ & 10 \\
\hline Deep Dermis & 1890 & 0 & $\mathrm{n} / \mathrm{a}$ & 4 \\
\hline
\end{tabular}

The optical coefficients applied to each layer of the skin model were based upon an analysis of the literature [17] (Figure 1). Absorption coefficients from Meglinski and Matcher's work [8] were applied alongside reduced scattering coefficients from Zonios et al [18]. The optical coefficients of vessel walls and blood filled lumina were obtained from Nishidate et al [19] and Friebel et al [20] respectively. 


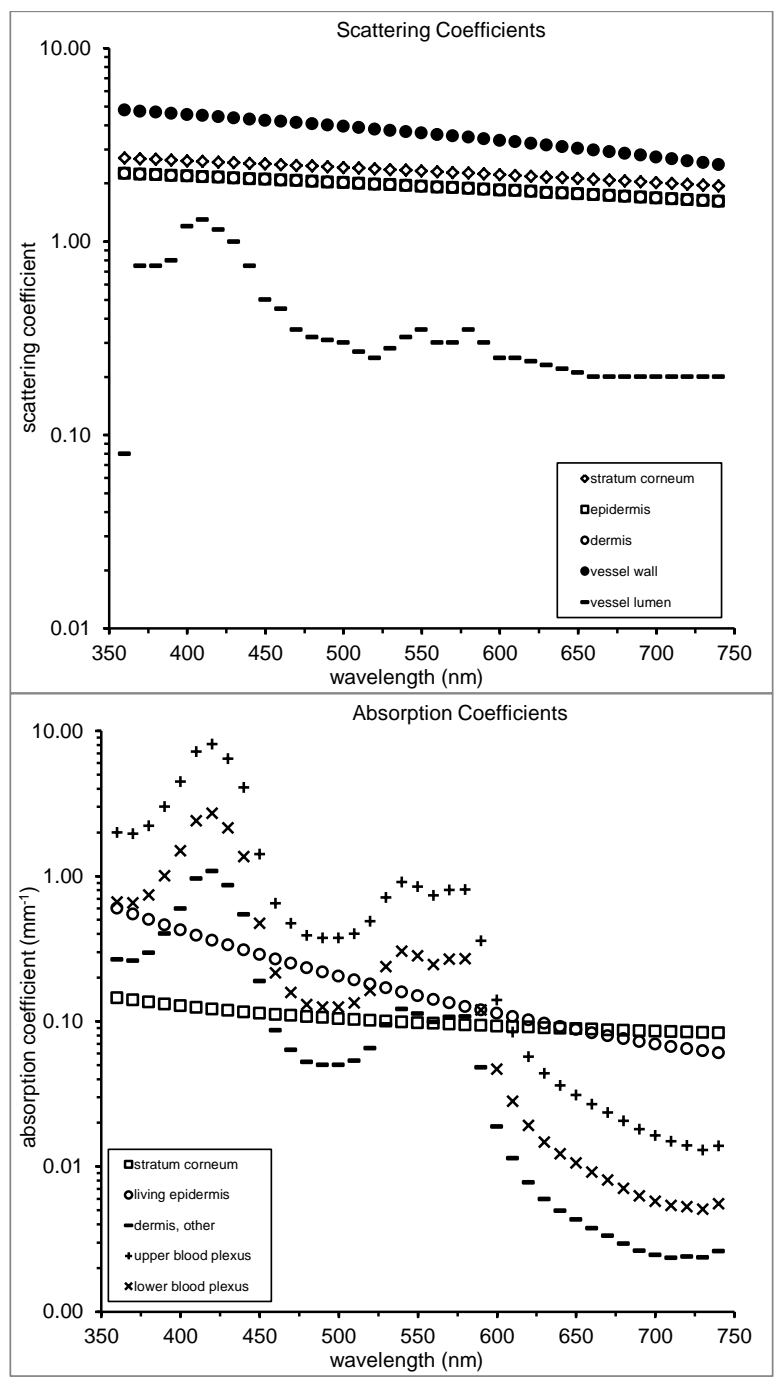

Figure 1: Scattering and absorption coefficients used in the skin model $[8,18]$.

Published reduced scattering coefficients $\left(\mu_{s}{ }^{\prime} \mathrm{mm}^{-1}\right)$ were converted to scattering coefficients $\left(\mu_{s} \mathrm{~mm}^{-1}\right)$ using the following relationship:

$$
\mu_{s}=\frac{\mu_{S}{ }^{\prime}}{1-g}
$$

Equation 1

where $g$ is the anisotropy factor. The anisotropy factors applied in this model were those introduced by van Gemert et al [21], and which have been used widely [19, 22-27]. Refractive indices from Ding et al [24] were applied individually to the stratum corneum, living epidermis and dermis. The refractive indices of blood vessel walls and lumina were obtained from Hui et al [28] and Keizjer et al [29]. 
The effect of melanosome diameter and number density upon the absorption and scattering coefficients of skin were considered in the mathematical model. The influence on absorption was simulated by adding a proportionate value of melanin absorption to the base epidermal absorption coefficient (Equation 2).

$$
\mu_{a}=\mu_{a}^{0}+C_{m e l} \mu_{a}^{m e l}
$$

Equation 2

where $C_{m e l}$ is the concentration (\%) of melanin within the epidermal layer, $\mu_{a}{ }^{m e l}$ is the absorption coefficient of melanin and $\mu_{a}^{0}$ is the absorption coefficient of the remaining epidermal layer. This is consistent with techniques applied elsewhere (for example, [8, 30-32]).

The degree of scatter caused by melanosomes within the skin is primarily dependent upon their size. The relationship between mean melanosome size and scattering strength has been investigated previously (Equation 3) [33]:

$$
\mu_{s}^{\text {melanin }}=\frac{32}{447} N_{A} \pi^{4} C V_{m} \frac{a^{3}}{\lambda^{4}}\left(\frac{\varepsilon-\varepsilon_{s}}{\varepsilon+2 \varepsilon_{s}}\right)^{2} \quad \text { Equation 3 }
$$

where $N_{A}$ is Avogadro's number $\left(6.02 \times 10^{23} \mathrm{~mol}^{-1}\right), C$ is the concentration of melanin (mol), $V_{m}=1.2 \times 10^{-28} \mathrm{~m}^{3}$ is described as the 'volume of a single monomer', $a$ is the mean melanosome size $(\mathrm{m})$, and $\varepsilon$ and $\varepsilon_{s}$ are the dielectric constants of melanin $\left(2.72 \mathrm{Fm}^{-1}\right)$ and water $\left(1.81 \mathrm{Fm}^{-1}\right)$ respectively. The new Monte Carlo programme considers Equation 3 in a simulation of skin optics for the first time.

A single layer of the skin model, whose superficial and deep extents were freely moveable, was introduced to the dermis to accommodate a simulated PWS lesion. Vessels, each consisting a wall and lumen, were created using the MATLAB cylinder command, producing a set of flat surfaces joined together to form a 10 sided open ended prism, oriented horizontally in the model (parallel to the skin surface) and extending beyond the model boundaries. The rand command was used to position and orientate the vessels with a uniform distribution throughout the layer. The maximum deviation in the $x$ and $y$ directions from the centre of the model was fixed at $3.5 \mathrm{~mm}$, ensuring that each vessel remained within the $8 \mathrm{~mm}$ diameter region illuminated during simulation. 


\section{Ray Tracing}

Light transport through the skin model was simulated using a RTT ray-tracing technique.

Figure 2 shows the basic layout of the programme:

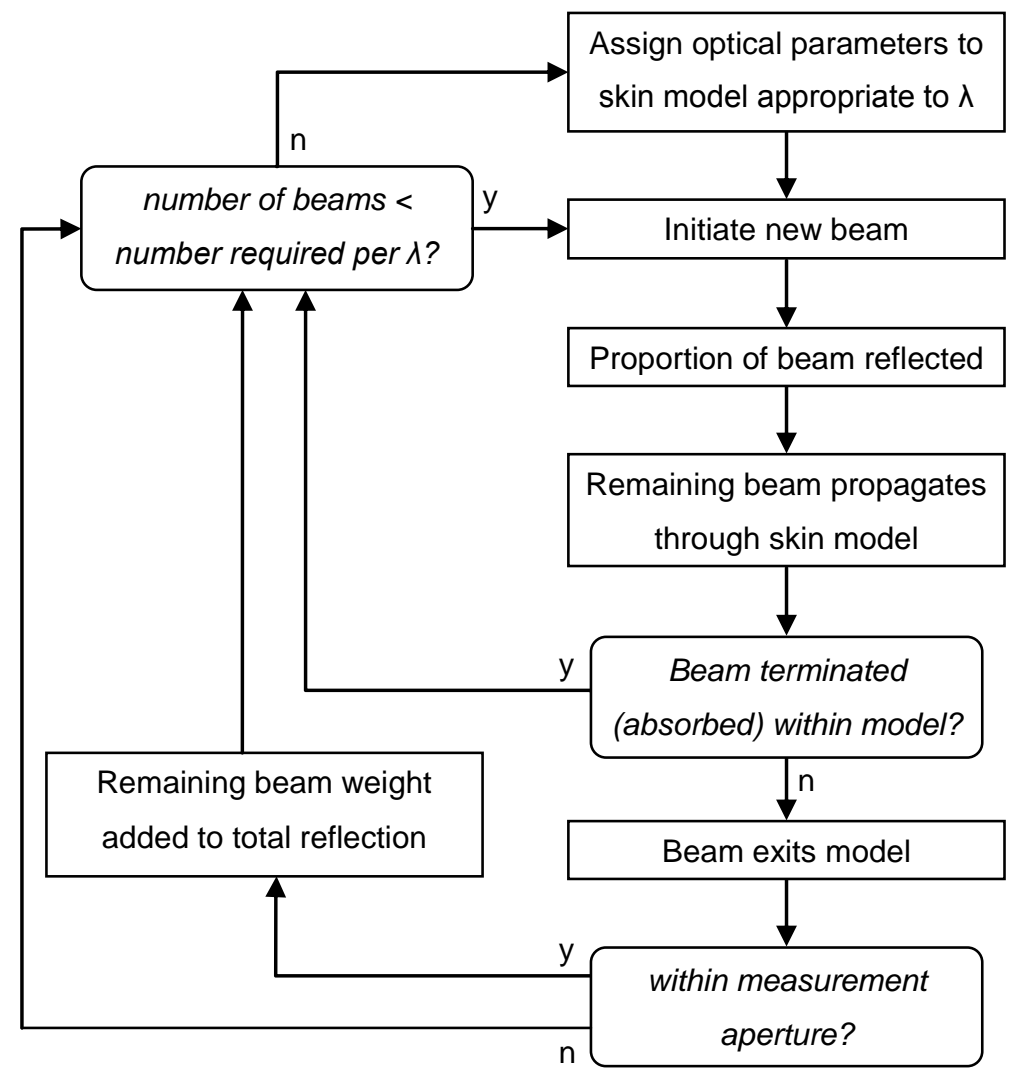

Figure 2: Flow chart showing basic structure of the new Monte Carlo program.

\section{Beam Initialisation}

Each ray, or beam, is initiated at the surface of the skin model within a circle of radius 4 $\mathrm{mm}$. Beam position and direction is allocated using the MATLAB rand command, which results in a uniform distribution across the aperture. This represents homogeneous illumination of the skin surface across the aperture using a perfectly diffuse illuminant. Each beam is attributed a weight of 1.0 upon initialisation. The beam weight is used for comparison between the total quantity of incident light energy and the total quantity of reflected and backscattered light energy at each wavelength.

Interactions at the Skin Surface 
Reflection is approximated using the Fresnel equations for an unpolarised source (Equation 4).

$$
R=\frac{R_{s}+R_{p}}{2}
$$

Equation 4

where: $R_{s}=\frac{\tan \left(\theta_{i}\right)-n_{s}(\lambda) \tan \left(\theta_{t}\right)}{\tan \left(\theta_{i}\right)+n_{s}(\lambda) \tan \left(\theta_{t}\right)} \& R_{p}=-\frac{\sin \left(\theta_{i}\right)-n_{s}(\lambda) \sin \left(\theta_{t}\right)}{\sin \left(\theta_{i}\right)+n_{s}(\lambda) \sin \left(\theta_{t}\right)}$

(Fresnel's Equations)

where $R_{s}$ is the proportion of $s$-polarised (perpendicular, or senkrecht polarised) light reflected and $R_{p}$ is the proportion of $p$-polarised (parallel polarised) light reflected (thus $R$, being the mean of these, is the proportion of unpolarised light reflected $\left.{ }^{1}\right), \vartheta_{i}$ and $\vartheta_{t}$ are respectively the incident and transmitted angles of the photon packet, $n_{s}(\lambda)$ is the refractive index of the skin. Surface reflection is simulated as a reduction in the beam weight by the proportion $R$ (Equation 21). The remaining beam is refracted as it enters the skin model. The degree of refraction is calculated using Snell's law (Equation 5).

$$
\theta_{t}=\arcsin \left(\frac{1}{n_{s}(\lambda)} \sin \left(\theta_{i}\right)\right)
$$

Equation 5

After propagating through the skin model, the beam may return to the skin surface. If this occurs within the $8 \mathrm{~mm}$ diameter aperture of the simulated acceptance window, then the transmitted beam weight is recorded as part of the total reflectance. A proportion of the beam is reflected back into the skin model as determined by the Fresnel equation (Equation 4) and the remaining (transmitted) beam is terminated.

\section{Simulating Scatter}

The mean distance between scattering events $(s)$ is determined using the RTT scattering coefficient ( $\mu_{s}$, Equation 6).

$$
s=\frac{-\log _{e}(\text { rand })}{\mu_{s}}
$$

Equation 6

\footnotetext{
${ }^{1}$ It is assumed that unpolarised light, as expected from a xenon flashlamp light source, does not have a greater amount of either $s$-polarised or $p$-polarised light.
} 
where rand is taken from a set uniformly distributed random numbers between 0 and 1 , created using the MATLAB rand command.

Non-boundary scattering is simulated in two stages:

1. The elevation angle (the angle between the beam direction and the $x-y$ plane) is adjusted in accordance with the modified Henyey-Greenstein equation [34]:

$$
H G=\frac{1-g^{2}}{\left[1+g^{2}-2 g \cdot \cos (2 \pi \cdot \text { rand })\right]^{3 / 2}}
$$

where $g$ is the anisotropy factor for the layer and wavelength in question.

2. The rotation angle (the angle between the $x$-axis and a projection of the beam along the $x-y$ plane) is adjusted isotropically:

$$
\theta^{\prime}=\theta+2 \pi \cdot \text { rand }
$$

Equation 8

where rand represents a value between 0 and 1 taken from a uniform distribution created using the MATLAB rand function.

\section{Simulating Absorption}

The RTT absorption coefficient defines the proportion of energy lost per unit path length through absorption effects. Absorption is simulated as a reduction in the beam weight, W:

$$
W=W e^{-\mu_{a} x}
$$

Equation 9

where $x$ is the path length. Equation 30 is applied at the end of each step, as well as at each interaction with a boundary (either layer boundaries, including the skin surface, or boundaries associated with simulated blood vessels). A proportion of beams whose weight drop below a predetermined value (0.001) are terminated using the previously reported roulette technique [29, 35-38].

\section{Layer Transitions}

When a beam transfers between layers of the skin model, or to or from a vessel wall or lumen, the reduction in beam weight is calculated and the remaining path length $\left(s_{\text {new }}\right)$ is 
adjusted in accordance with the scattering coefficient of the new layer or structure $\left(\mu_{s}^{\text {new }}\right)$, as follows:

$$
s_{\text {new }}=(s-p) \frac{\mu_{s}^{\text {old }}}{\mu_{s}^{\text {new }}}
$$

where $p$ is the distance along the beam path between the previous interaction or transition and the current transitional point. Further to this, the direction of the beam may be adjusted where a change in refractive index occurs. The new direction is calculated using Snell's Law.

Termination of a beam may occur independently of the roulette procedure if a beam exits the skin model at the skin surface, and at the remaining boundaries of the skin model. These extend to $100 \mathrm{~mm}$ in the horizontal directions and $40 \mathrm{~mm}$ in depth.

\section{Output}

The simulated spectral reflection consists of 39 points over the visible spectrum from 360 $\mathrm{nm}$ to $740 \mathrm{~nm}$ (in $10 \mathrm{~nm}$ steps). Each point represents the sum of simulated beam weight reflected from the surface and returning from the model volume within the $8 \mathrm{~mm}$ diameter aperture. These data points are presented as a proportion of the total beam weight entering the model, and thus represents measured reflectance as would be determined using an integrating sphere and a spectrally uniform, perfectly diffuse light source.

\section{Minimisation}

Two new minimisation procedures were developed for the interpretation of colour measurement data. The first procedure adjusts the melanin properties within the skin model such that the simulated reflectance spectrum may be minimised to the measured spectral reflectance of non-lesional skin. The second procedure builds upon this, adding blood vessels and adjusting their properties in order that the simulated curve may be minimised to a colour measurement of PWS skin from the same individual (Figure 3). 


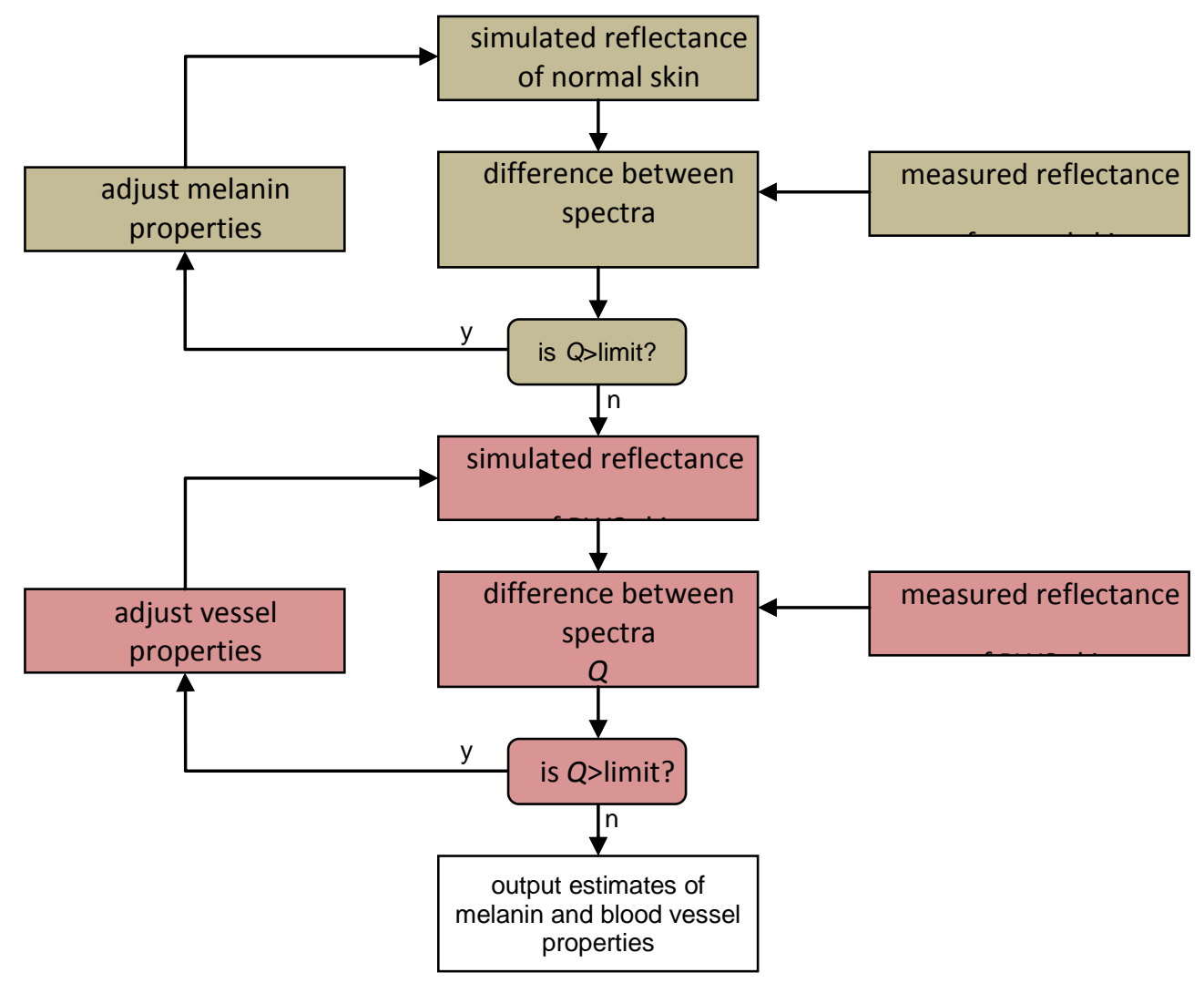

Figure 3: Outline of the minimisation procedure developed in the New Monte Carlo program.

\section{Melanin Adjustment}

Melanin granule diameter and volume fraction were adjusted proportionately across the three epidermal layers. During minimisation, stratum corneum granule diameter was limited to a minimum of $1 \mathrm{~nm}$ and a maximum of $20 \mathrm{~nm}[14,16]$ whilst melanosome size in the living epidermis was allowed to vary from $30 \mathrm{~nm}$ to $400 \mathrm{~nm}$ [12-15].

Melanin volume fraction was allowed to vary between $0 \%$ and $10 \%$ for each layer, although effectively this limit only applied to the deep living epidermis whose melanin content was greatest. Adjustment of the simulated mean diameter of melanosomes within the skin model was performed independently of melanin volume fraction. During diameter adjustment, only the scattering coefficients were changed, and the effects of melanosome diameter on the absorption coefficients were ignored.

In order to achieve robust minimisation when adjusting melanin diameter and concentration parameters, the total difference between the measured and simulated 
spectra was divided into two sections. The parameter $q m e l_{1}$ represents the total difference at the blue end of the spectrum and $\left.q_{m e}\right|_{2}$, the total difference at the red end. If both parameters showed a simulated curve sufficiently greater than the measured curve, then the melanin concentration was increased. Similarly, if both parameters showed a simulated curve sufficiently less than the measured curve, then the melanin concentration was decreased. However, if qmel $_{1}$ showed that the simulated curve was sufficiently greater or less than the measured curve but qmel $_{2}$ was not, then the melanin diameter was decreased or increased respectively.

To improve the rate of convergence whilst maintaining precision, limits were set for these parameters whereby large deviations in melanin concentration or diameter were instigated if the difference between simulated and measured reflectance curves were great, and smaller deviations were carried out for small differences.

\section{Blood Vessel Adjustment}

In accordance with the anticipated effects of adjusting PWS lesion depth, vessel diameter and vessel number, the spectrum was split into three regions (qves $\left.{ }_{1}-q v e s_{3}\right)$. Parameter adjustment was carried out as follows:

- If the simulated reflection differed greatly from the measured PWS skin sample over the majority of the spectrum (qves ${ }_{1}$ ) then the number of vessels was adjusted.

- Otherwise, if the simulated reflectance was less than the measured reflectance over the majority of the spectrum (qves ${ }_{1}$ ) then the depth of the simulated vessels was reduced, and vice-versa.

- Or if the simulated spectrum was very much greater than the measured spectrum at the red end of the spectrum (qves 3 ) relative to the blue/green end $\left(q v e s_{2}\right)$, then vessel diameter was increased. Conversely, if qves $_{2}$ was much larger than qves, then vessel diameter was decreased.

- Otherwise, if qves $_{1}$ or qves $_{2}$ still showed enough of a discrepancy, the vessel number was adjusted accordingly. 
This minimisation technique was designed to ensure fast convergence whilst maximising the probability of obtaining a realistic, unique and repeatable solution for any individual measurement of PWS skin colour.

Colour measurements were carried out on PWS and adjacent non-PWS skin of 10 participants using a Konica-Minolta CM2600-d integrating sphere spectrophotometer. The absolute maximum deviation from the mean across the measured spectrum for 50 consecutive measurements of a fixed object was $0.08 \%$, and on the inner forearm of the author was $1.4 \%$ measured reflection for this device, demonstrating a high level of repeatability consistent with previous studies [39].

\section{Results and Discussion}

An initial simulation of spectral reflectance was produced from the new Monte Carlo programme using the parameters derived from the literature (Figure 1). Figure 4 shows the simulated spectral reflectance alongside the mean of 5 consecutive measurements of non-lesional skin colour obtained from 10 participants.

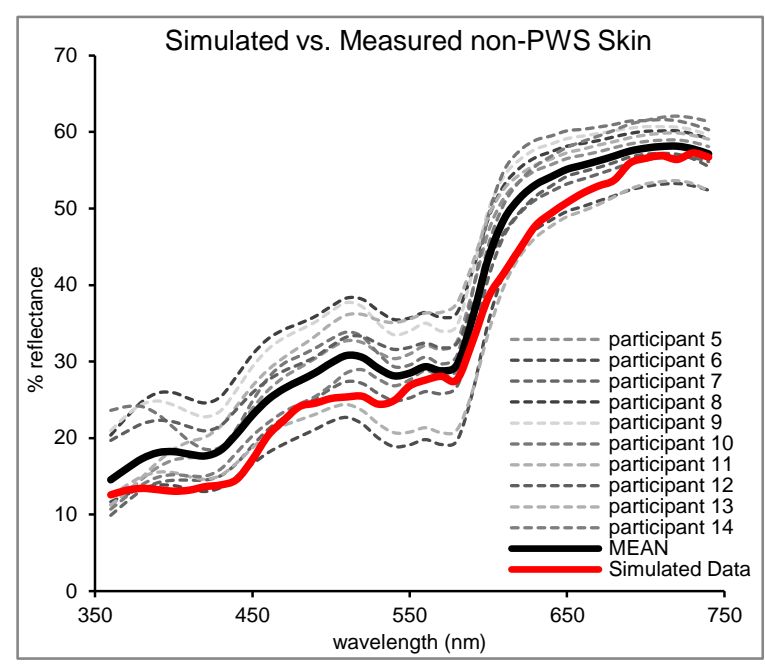

Figure 4: Comparison of simulated reflectance obtained from published data and measured skin reflectance from the participants of this study.

Overall, the simulated spectrum shows similar characteristics to the average measured curve and all of the data points sit within the range of measured spectra. 


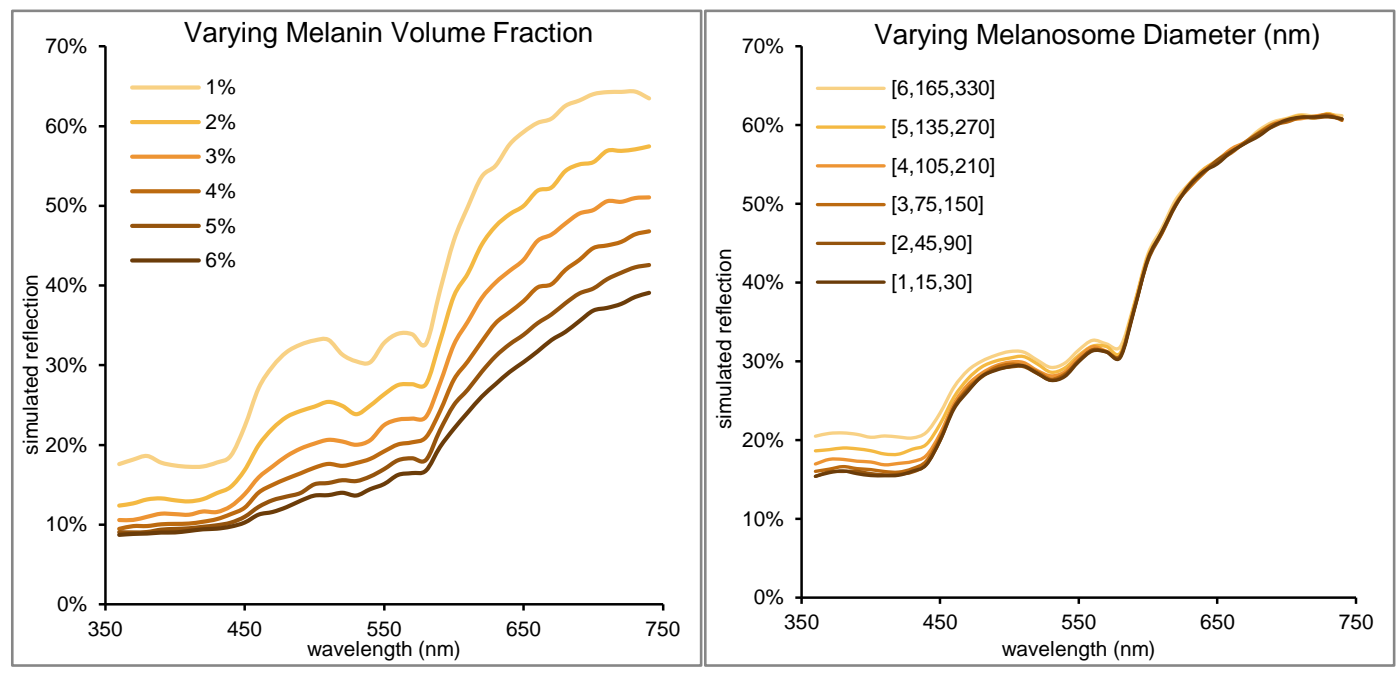

Figure 5: The effect of varying mean melanosome volume fractions (left) and diameters (right) upon the simulated reflectance spectrum (simulated diameters for [stratum corneum, superficial epidermis, dermis] given in $\mathrm{nm}$.

The simulated effect of epidermal melanin within the skin model was assessed by increasing the mean epidermal content in $1 \%$ steps (weighted across the three layers according to volume; Figure 5 , left). Increasing from a mean of $1 \%$ to $2 \%$ epidermal melanin volume resulted in a near uniform decrease in simulated reflection. Further increases in melanin content had a greater effect towards the red end of the spectrum and also resulted in a flatter simulated reflectance curve. Also, with each $1 \%$ increase in epidermal melanin volume, the overall change in simulated reflectance was less.

Increasing the diameter of melanosomes within the skin model had minimal effect in the region from $600 \mathrm{~nm}$ to $750 \mathrm{~nm}$. The most noticeable increase in simulated reflectance occurred in the region from $360 \mathrm{~nm}$ to $450 \mathrm{~nm}$ (Figure 5, right).

\section{Minimisation Procedure - Melanin Adjustment}

Based upon the preliminary results presented in Figure 5, the following regions were selected: $360-540 \mathrm{~nm}$ for qmel1, used to inform melanosome diameter and 640-740 nm for qmel2, which is used to inform the quantity of epidermal melanin. The melanin minimisation was tested using a skin model representative of type II Northern European skin (1.2\% mean epidermal melanin volume, melanosome diameters of $30 \mathrm{~nm}$ in the deep living epidermis, $15 \mathrm{~nm}$ in the superficial living epidermis and $1 \mathrm{~nm}$ in the stratum corneum). The simulated output was minimised to the mean spectrum from the nonlesional skin of 10 participants (Figure 6). 

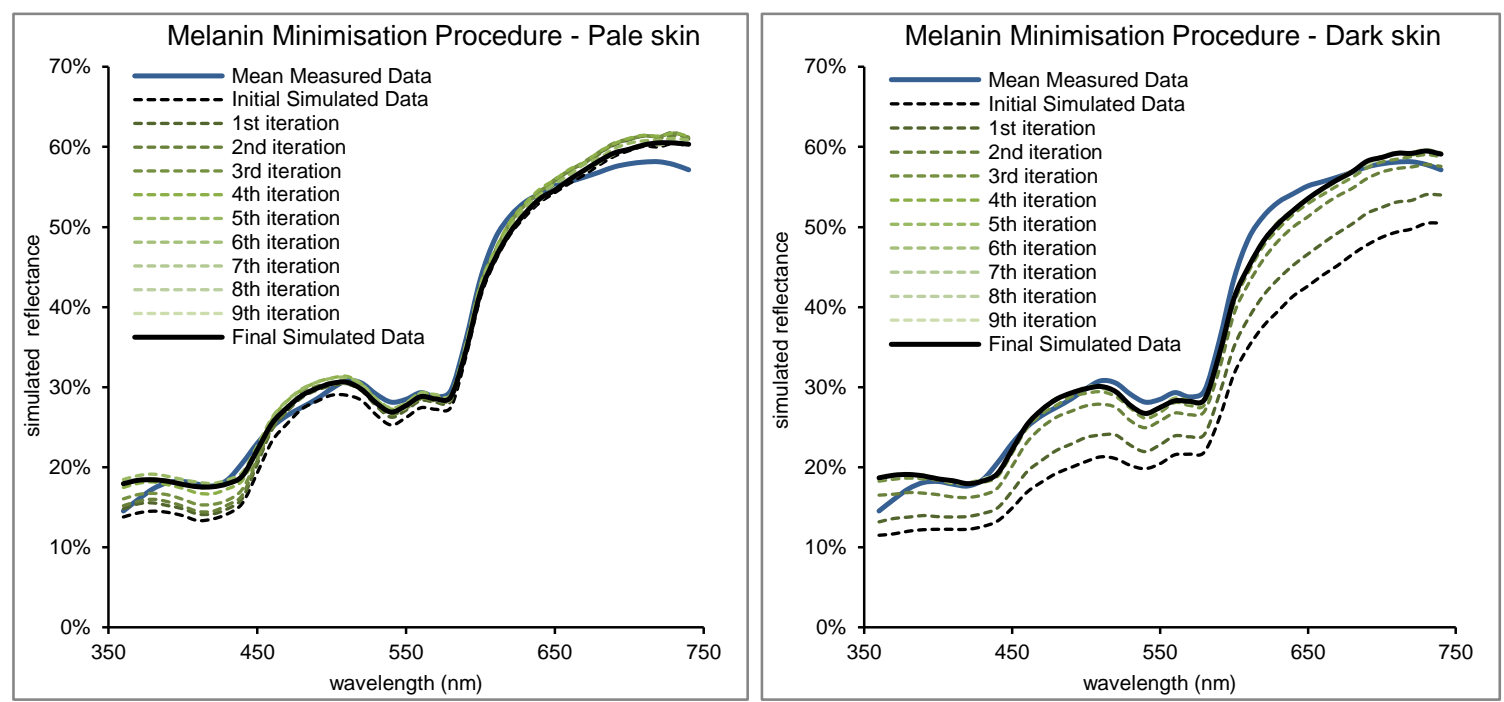

Figure 6: Simulated spectral reflection outputs obtained during verification of the melanin minimisation procedure starting from melanin parameters representative of light (left) and dark (right) skin types.

The initial simulated data showed a close resemblance to the mean measured dataset.

The minimisation procedure was successful in improving this fit over the majority of the spectrum, although the final fit was not improved at wavelengths greater than $600 \mathrm{~nm}$ (Figure 6, left). This is due to the smoothly varying spectral absorption and scattering properties of melanin, which do not allow for independent variation of simulated reflectance in this region.

To obtain a better understanding of the rate of convergence and the repeatability of the procedure, a further simulation was carried out using a different initial dataset. In this case, the procedure began with a skin model considered to be representative of dark Afro-Caribbean (type V) skin ( $5 \%$ epidermal melanin volume, melanosome diameters of $300 \mathrm{~nm}$ in the deep living epidermis, $150 \mathrm{~nm}$ in the superficial living epidermis and $10 \mathrm{~nm}$ in the stratum corneum, Figure 6, right).

It can be seen from Figure 6 that the initial simulated reflectance differed substantially from the measured dataset. Convergence was fast, resulting in good agreement between simulated and measured spectra after only 3 iterations of the minimisation procedure. The procedure, involving 11 iterations for each experiment, took an average of 28 hours to perform over the two validation experiments, using a quad-core processer with $16 \mathrm{~Gb}$ of RAM and a nominal clock rate of $2.66 \mathrm{GHz}$. 
Table 2: Final prediction of melanin characteristics for the mean participant dataset determined from two different initial skin models.

\begin{tabular}{|cr|r|r|}
\hline \multicolumn{2}{|c|}{ Estimated epidermal melanin characteristics } & \multicolumn{1}{c|}{ Type II } & \multicolumn{1}{c|}{ Type V } \\
\hline \multirow{2}{*}{ melanosome volume } & stratum corneum & $0.5 \%$ & $0.2 \%$ \\
fraction & superficial living epidermis & $1.0 \%$ & $1.0 \%$ \\
& deep living epidermis & $2.0 \%$ & $2.4 \%$ \\
\hline \multirow{2}{*}{ mean melanosome } & stratum corneum & $11 \mathrm{~nm}$ & $11 \mathrm{~nm}$ \\
diameter & superficial living epidermis & $165 \mathrm{~nm}$ & $165 \mathrm{~nm}$ \\
& deep living epidermis & $330 \mathrm{~nm}$ & $330 \mathrm{~nm}$ \\
\hline
\end{tabular}

The predicted melanin volume fractions are consistent with values published elsewhere for Northern European skin types [11]. Although the spread of melanin through the simulated epidermis differed due to the differences in the initial skin models, the final prediction from the two experiments were similar overall (Table 2). The melanosome diameters predicted from the two experiments were identical, although they were greater than expected these skin types [12-16].

\section{Adjusting PWS Vessel Properties}

The effect of changing lesion depth was assessed in the simulation by introducing 10 vessels of $20 \mu \mathrm{m}$ diameter and varying their mean depth within the model.

Increasing the depth of the lesion within the skin model was shown to cause a broadband increase in simulated reflectance (Figure 7, bottom left). The increase in reflectance was less with each $100 \mu \mathrm{m}$ step, as the proportion of light reaching the vessels decreased. This effect is particularly evident between $400 \mathrm{~nm}$ and $450 \mathrm{~nm}$, where scattering is high (penetration depth is low) but absorption by blood is lower than neighbouring wavelength regions. 

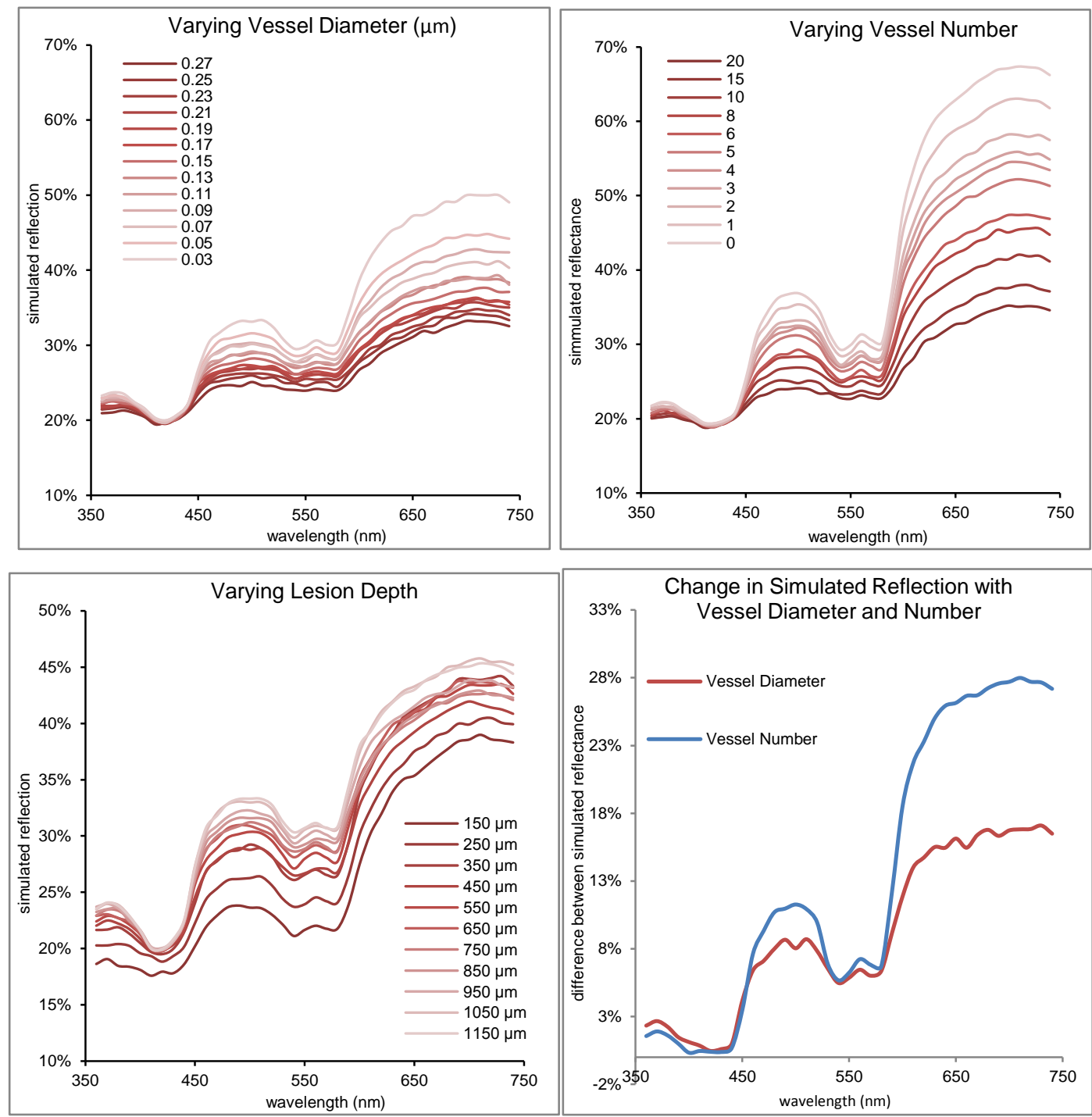

Figure 7: Simulated reflectance for vascular lesions of: (top left) 10 vessels at a mean depth of $500 \mu \mathrm{m}$, and vessel diameters varying from 0.03-0.27 $\mu \mathrm{m}$, (top right) fixed mean vessel diameter $(0.03 \mathrm{~mm}$ ), lesion depth and layer thickness, but number of simulated PWS vessels varying from 0 to 12 , (bottom left) fixed vessel number, mean diameter and layer thickness, but mean depth varying from $150 \mu \mathrm{m}$ to $1150 \mu \mathrm{m}$. (Bottom right) Absolute change in simulated reflectance resulting from change in vessel number from 1-20 (blue), with a fixed diameter of $30 \mu \mathrm{m}$, and from a change in vessel diameter from $30 \mu \mathrm{m}-270 \mu \mathrm{m}$ with a fixed number of 10 vessels.

Increasing the diameter of vessels was shown to increase simulated reflection across the spectrum. This effect is greatest at longer wavelengths, in particular at wavelengths longer than $630 \mathrm{~nm}$ (Figure 7, top left). The effect is less pronounced at the haemoglobin absorption peaks, where the effective path length within the vessel is shortest and the albedo is smallest, in particular near $420 \mathrm{~nm}$. Varying vessel number had a broad effect, 
but was most prominent in the regions from $450-530 \mathrm{~nm}$ and $630-740 \mathrm{~nm}$ (Figure 7, top right).

On first inspection, the characteristics of varying vessel number appear to mirror those of vessel diameter. However, the effect of varying each parameter independently is shown in more detail in Figure 7, bottom right. Here, a similar change in simulated reflectance is induced in the regions $360-460 \mathrm{~nm}$ and $540-570 \mathrm{~nm}$ by adjusting either vessel number from 1-20 at a fixed diameter of $30 \mu \mathrm{m}$, or vessel diameter between $30 \mu \mathrm{m}$ and $270 \mu \mathrm{m}$ at a fixed number of 10. A much stronger response results from adjusting vessel number relative to vessel diameter in the remaining wavelength regions. Thus, for a fixed epidermis, due to the difference in their contribution to spectral changes in simulated reflectance, any combination of vessel number, diameter and depths will result in a unique simulated output using the new Monte Carlo programme.

Minimisation Procedure - Vessel Adjustment

Verification of the PWS vessel minimisation procedure was carried out in a similar manner to the melanin minimisation procedure validation. PWS vessel diameters were limited from a minimum of $20 \mu \mathrm{m}$ to a maximum of $500 \mu \mathrm{m}$. Based upon the preliminary results presented in Figure 7, the following regions were selected:

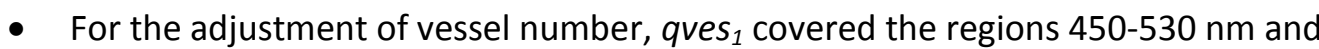
$630-740 \mathrm{~nm}$.

- For the adjustment of vessel depth, qves $_{2}$ covered the entire wavelength region, from $340-740 \mathrm{~nm}$.

- For the adjustment of vessel diameter, qves ${ }_{3}$ covered the region 530-590 nm.

Due to overlap of the region qves ${ }_{2}$ with qves ${ }_{1}$ and qves $_{3}$, adjustment of lesion depth is performed independently of vessel number and diameter. This required an iterative technique, whereby vessel number and diameter were adjusted initially followed by lesion depth, before vessel number and diameter were again considered.

Starting with the final parameters from the melanin minimisation procedure (Figure 6), the simulated spectrum was minimised to the mean measured spectral reflectance of PWS skin from the same 10 participants over twelve iterations (Figure 44). 


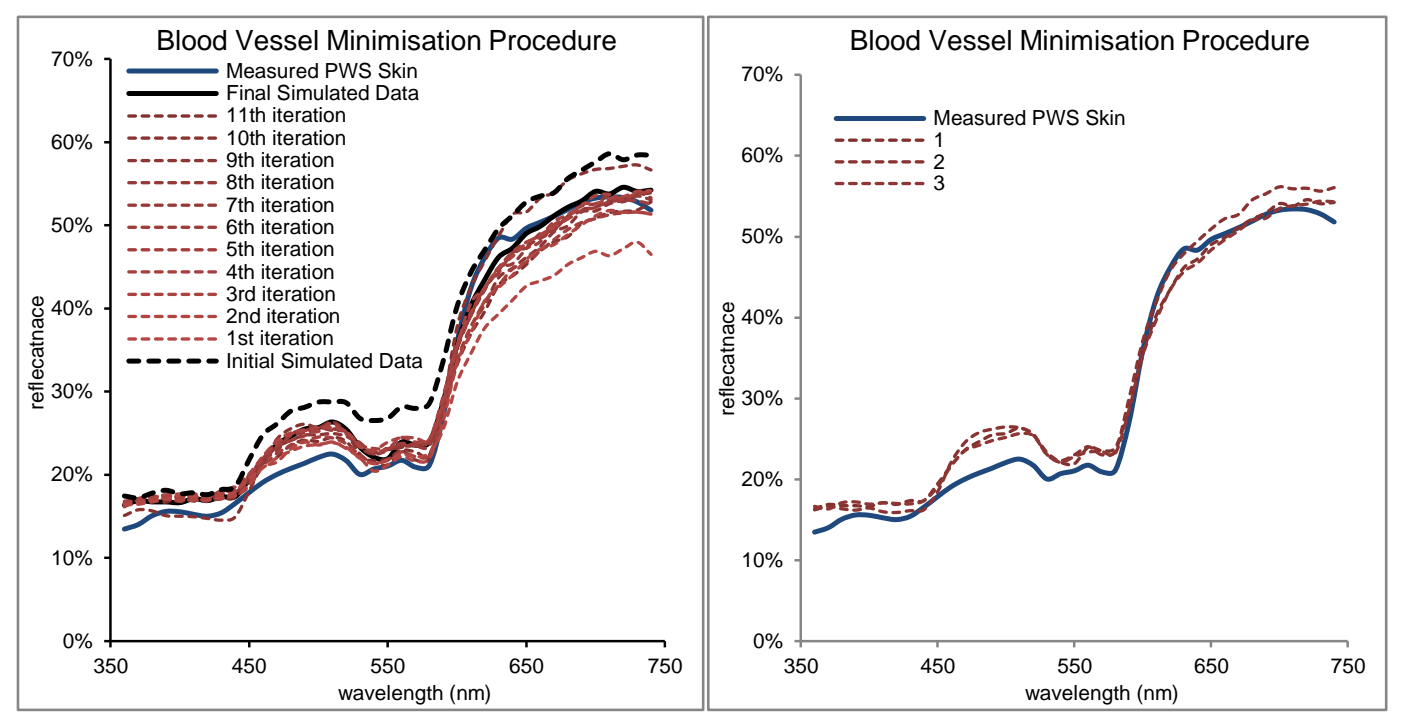

Figure 8: [left] Simulated spectral reflection outputs obtained during verification of the blood vessel minimisation procedure. [right] Repeated outputs from blood vessel minimisation procedure showing consistency of minimisation procedure after 12 iterations.

The final simulated reflection shown in Figure 8 provided a close fit to the measured spectrum, with a mean deviation of less than $2 \%$ reflection. An excellent fit was produced in the region $540-740 \mathrm{~nm}$ (mean 1.3\% deviation), although there was greater deviation from the measured dataset in the region from $460 \mathrm{~nm}-540 \mathrm{~nm}$ (mean 3.3\% deviation). This was obtained using a skin model containing 7 vessels with an outside vessel diameter of $70 \mu \mathrm{m}$ and a mean depth of $160 \mu \mathrm{m}$. The preliminary work presented in Figure 7 suggest that a closer fit may have been produced using a skin model with slightly larger, more superficial (and therefore fewer) vessels.

The procedure was repeated on two occasions with the same input parameters and produced comparable results after 12 iterations ( 8 vessels of $70 \mu \mathrm{m}$ diameter at a mean depth of $210 \mu \mathrm{m}$, and 7 vessels of $80 \mu \mathrm{m}$ diameter at a mean depth of $180 \mu \mathrm{m}$, see Figure 45). The blood vessel procedure took a mean time of approximately 4.5 days using a quad-core processer with $16 \mathrm{~GB}$ of RAM and a nominal clock rate of $2.66 \mathrm{GHz}$ (in addition to 1 day for the melanin procedure).

\section{Analysis}

The determination of appropriate skin optical coefficients is challenging. Further to this, the use of a simple skin model consisting of a perfectly smooth surface, homogeneous 
layers and a simulated light source that was both homogeneous and perfectly diffuse is not truly representative of the complex and varied nature of human skin, or the imperfections found in any skin colour measurement setup. Despite these challenges, skin optical coefficients determined elsewhere applied to the model presented here produced a spectral reflectance curve, which compared well with the measured reflection from the ten participants selected for this study.

In comparison to the mean measured data, the simulated curve demonstrated less reflectance over the entire spectrum. This may be due to differences in skin type between the participant group measured and those informing published data. The population demographic of the participant group in this study is typical of Salisbury, UK and its surrounding areas and consists mainly of individuals with pale, Northern European skin types. The published data used to inform the simulation presented in Figure 1 is sourced from institutions based in the UK [8], Greece [32] and The Netherlands [21] and, although not explicitly stated, it is likely that these may include slightly darker skin types on average.

Adjusting melanin volume fractions and simulated melanosome diameters within the skin model each had a significant but distinctive effect upon the simulated spectral reflectance. Due to the broadband absorption spectrum of melanin, changes in melanosome volume fraction resulted in changes in reflectance across the visible spectrum. However, due to the wavelength ${ }^{-4}$ dependence of scatter on melanosome diameter in the model, the effect on simulated reflection was found to be negligible at the red end of the spectrum. As a result of these differences, independent adjustment of each parameter allows for an adequate degree of flexibility for reproducing measured spectral reflectance curves whilst maintaining a unique output for any available combination of these two melanin parameters.

The convergence rate of the melanin minimisation procedure was fast and resulted in realistic predictions of melanin volume fractions for this participant group. The melanosome diameters predicted by the simulation were greater than expected. Figure 6 and Figure 8 show that simulated spectral reflectance was greater than measured values 
at the violet end of the spectrum, suggesting that a more refined minimisation procedure would have predicted smaller (and therefore more realistic) melanosome diameters.

The effects of changing vessel depth, diameter and number density were each found to produce distinct changes in the simulated reflectance of PWS skin. This is in agreement with previous biopsy $[3,4]$ and simulation $[26,40]$ studies. As a result, any combination of these three vessel parameters, for a fixed epidermis, creates a unique spectral reflectance curve.

The blood vessel minimisation procedure is more complex than the equivalent melanin procedure, resulting in a slower convergence rate. However, the blood vessel parameters predicted by the procedure were shown to be consistent with those found elsewhere in the literature $[3,4]$ and resulted in spectral reflectance curves, which reproduced measured spectra to a satisfactory degree of accuracy. It is likely that further iterations of the procedure would have resulted in a closer fit between measured and simulated spectra, as well as improved repeatability in the predicted vessel parameters. The number of iterations is limited by the computational expense, and therefore the length of time taken to complete each iteration.

Thus, in order to improve the usability of this method within the clinic, processing times need to be improved. Computational time for this simulation will decrease with the availability of faster processing speeds. Further to this, techniques involving programming onto computing graphic cards have been shown to vastly reduce processing times in Monte Carlo simulations of skin optics [41]. Such techniques will allow for more iterations and may one day bring allow such a procedure to be used in the clinic.

\section{Conclusions}

A new Monte Carlo programme was produced to simulate spectral reflectance and, through minimisation to a measured dataset from an individual PWS lesion, predict the mean depth, diameter and number density of PWS vessels.

Initial results demonstrate a good fit between measured and simulated spectral reflection although the process is computationally expensive. The accuracy of the predicted vessel characteristics for this participant group cannot be verified directly as the true vessel 
characteristics are not known, although predictions are in line with the range of parameters found elsewhere. Further work comparing predicted parameters to known values is necessary to further determine the efficacy of this technique. 


\section{References}

1. Nelson JS, Milner TE, Tanenbaum BS, et al. Infra-red tomography of port-wine-stain blood vessels in human skin. Lasers in Medical Science, 1996, 11(3): 199-204.

2. van Gemert MJC, Nelson JS, Milner TE, et al. Non-invasive determination of port wine stain anatomy and physiology for optimal laser treatment strategies. Physics in Medicine and Biology, 1997, 42(5): 937-950.

3. Barsky SH, Rosen S, Geer DE, et al. Nature and evolution of port wine stains computer-assisted study. Journal of Investigative Dermatology, 1980, 74(3): 154-157.

4. Fiskerstrand EJ, Svaasand LO, Kopstad G, et al. Laser treatment of port wine stains: Therapeutic outcome in relation to morphological parameters. British Journal of Dermatology, 1996, 134(6): 1039-1043.

5. Le KVT, Shahidullah $\mathrm{H}$ and Frieden IJ. Review of modern techniques in detecting port-wine stain response to laser therapy. Dermatologic Surgery, 1999, 25(2): 127-132.

6. Pickering JW, Mordon SR and Brunetaud JM. The objective reporting of laser treatment of port wine stains. Lasers in Medical Science, 1992, 7(4): 415-421.

7. Reynolds N, Exley J, Hills S, et al. The role of the lumina intense pulsed light system in the treatment of port wine stains - a case controlled study. British Journal of Plastic Surgery, 2005, 58(7): 968-980.

8. Meglinski IV and Matcher SJ. Quantitative assessment of skin layers absorption and skin reflectance spectra simulation in the visible and near-infrared spectral regions. Physiological Measurement, 2002, 23(4): 741-753.

9. Braverman IM. The cutaneous microcirculation: Ultrastructure and microanatomical organization. Microcirculation-London, 1997, 4(3): 329-340.

10. Moretti G, Ellis R and Mescon H. Vascular patterns in the skin of the face. Jour Invest Dermatol, 1959, 33((3)): 103-112.

11. Rawlings AV. Ethnic skin types: Are there differences in skin structure and function? International Journal of Cosmetic Science, 2006, 28(2): 79-93.

12. Bashkatov AN, Genina EA, Kochubey VI, et al. Optical properties of melanin in the skin and skin-like phantoms. Proceedings of the SPIE - The International Society for Optical Engineering, 2000, 4162(219-226.

13. Minwalla L, Zhao Y, Le Poole IC, et al. Keratinocytes play a role in regulating distribution patterns of recipient melanosomes in vitro. Journal of Investigative Dermatology, 2001, 117(2): 341-347.

14. Selby CC. An electron microscope study of the epidermis of mammalian skin in thin sections. I. Dermo-epidermal junction and basal cell layer. The Journal of biophysical and biochemical cytology, 1955, 1(5): 429-444.

15. Thong HY, Jee SH, Sun CC, et al. The patterns of melanosome distribution in keratinocytes of human skin as one determining factor of skin colour. British Journal of Dermatology, 2003, 149(3): 498-505.

16. Watt AAR, Bothma JP and Meredith P. The supramolecular structure of melanin. Soft Matter, 2009, 5(19): 3754-3760.

17. Lister T, Wright PA and Chappell PH. Optical properties of human skin. Journal of Biomedical Optics, 2013, 17(9):

18. Zonios $\mathrm{G}$ and Dimou A. Light scattering spectroscopy of human skin in vivo. Optics Express, 2009, 17(3): 1256-1267. 
19. Nishidate I, Aizu Y and Mishina H. Depth visualization of a local blood region in skin tissue by use of diffuse reflectance images. Optics Letters, 2005, 30(16): 2128-2130.

20. Friebel M, Helfmann J, Muller $\mathrm{G}$, et al. Influence of shear rate on the optical properties of human blood in the spectral range 250 to $1100 \mathrm{~nm}$. Journal of Biomedical Optics, 2007, 12(5): 054005-054005.

21. van Gemert MJC, Jacques SL, Sterenborg H, et al. Skin optics. leee Transactions on Biomedical Engineering, 1989, 36(12): 1146-1154.

22. Bruls WAG and Vanderleun JC. Forward scattering properties of human epidermal layers. Photochemistry and Photobiology, 1984, 40(2): 231-242.

23. Graaff R, Aarnoudse JG, Zijp JR, et al. Reduced light-scattering properties for mixtures of spherical-particles - a simple approximation derived from mie calculations. Applied Optics, 1992, 31(10): 1370-1376.

24. Ding H, Lu JQ, Wooden WA, et al. Refractive indices of human skin tissues at eight wavelengths and estimated dispersion relations between 300 and $1600 \mathrm{~nm}$. Physics in Medicine and Biology, 2006, 51(6): 1479-1489.

25. Jacques SL. Origins of tissue optical properties in the uva, visible, and nir regions. OSA Trends in Optics and Photonics on Advances in Optical Imaging and Photon Migration. Vol.2 From the Topical Meeting, 1996, 364-371.

26. Svaasand LO, Norvang LT, Fiskerstrand EJ, et al. Tissue parameters determining the visual appearance of normal skin and port-wine stains. Lasers in Medical Science, 1995, 10(1): 55-65.

27. Verkruysse W, Pickering JW, Beek JF, et al. Modeling the effect of wavelength on the pulsed dye-laser treatment of port wine stains. Applied Optics, 1993, 32(4): 393-398.

28. Hui L, Lei $L$ and Shusen $X$. Refractive index of human whole blood with different types in the visible and near-infrared ranges. Proceedings of the SPIE - The International Society for Optical Engineering, 2000, 3914(517-521.

29. Keijzer M, Jacques SL, Prahl SA, et al. Light distributions in artery tissue - montecarlo simulations for finite-diameter laser-beams. Lasers in Surgery and Medicine, 1989, 9(2): 148-154.

30. Donner C, Weyrich $\mathrm{T}$, d'Eon $\mathrm{E}$, et al. A layered, heterogeneous reflectance model for acquiring and rendering human skin. Acm Transactions on Graphics, 2008, 27(5):

31. Maeda T, Arakawa N, Takahashi M, et al. Monte carlo simulation of spectral reflectance using a multilayered skin tissue model. Optical Review, 17(3): 223-229.

32. Zonios G, Bassukas I and Dimou A. Comparative evaluation of two simple diffuse reflectance models for biological tissue applications. Applied Optics, 2008, 47(27): 49654973.

33. Riesz J. The spectroscopic proerties of melanin. PhD Thesis, University of Queensland, 2007.

34. Jacques SL, Alter CA and Prahl SA. Angular dependence of hene laser light scattering by human dermis. Lasers in the Life Sciences, 1987, 1(4): 309-333.

35. Nishidate I, Maeda T, Aizu Y, et al. Visualizing depth and thickness of a local blood region in skin tissue using diffuse reflectance images. Journal of Biomedical Optics, 2007, 12(5):

36. Prahl SA, Keijzer M, Jacques SL, et al. A monte carlo model of light propagation in tissue. SPIE Proceedings of Dosimetry of Laser Radiation in Medicine and Biology, 1989,

5(102-111. 
37. Wang L, Jacques SL and Liqiong Z. Mcml-monte carlo modeling of light transport in multi-layered tissues. Computer Methods and Programs in Biomedicine, 1995, 47(2): 131146.

38. Yaroslavsky AN, Yaroslavsky IV, Goldbach T, et al. Influence of the scattering phase function approximation on the optical properties of blood determined from the integrating sphere measurements. Journal of Biomedical Optics, 1999, 4(1): 47-53.

39. Lister TS, Wright PA and Chappell PH. Spectrophotometers for the clinical assessment of port-wine stain skin lesions: A review. Lasers in Medical Science, 2010, 25(3): 449-457.

40. Verkruysse W, Lucassen GW and van Gemert MJC. Simulation of color of port wine stain skin and its dependence on skin variables. Lasers in Surgery and Medicine, 1999, 25(2): 131-139.

41. Alerstam E, Lo WCY, Han TD, et al. Next-generation acceleration and code optimization for light transport in turbid media using gpus. Biomedical Optics Express, 2010, 1(2): 658-675. 